\title{
Cohetes de aire: construcción, fundamentos y aplicaciones didácticas para el estudio de la Física en bachillerato y secundaria
}

\author{
Antonio Tomás-Serrano \\ IESVega del Táder. Molina de Segura (Murcia).España.atserrano@gmail.com \\ ORCID: bttp:/ / orcid.org/0000-0002-4325-6562 \\ Jerónimo Hurtado-Pérez \\ Museo de Ciencias MUDIC. Oribuela (Alicante).España.jeronimoh@gmail.com \\ ORCID: bttp:/ / orcid.org/0000-0002-1301-3201
}

[Recibido: 12 Mayo 2019. Revisado: 4 Julio 2019. Aceptado: 7 Julio 2019]

\begin{abstract}
Resumen: Los cohetes impulsados por aire comprimido son menos conocidos que los cohetes de agua, pero tienen un alcance similar, son más sencillos de construir y no presentan riesgos, ni en su manipulación ni en su lanzamiento, ya que la energía necesaria para impulsarlos puede conseguirse simplemente al presionar con el pie un recipiente flexible lleno de aire. Las actividades que se pueden desarrollar con estos cohetes son adaptables tanto para alumnos de enseñanza secundaria obligatoria como para bachillerato y admiten múltiples enfoques, en función del nivel del alumnado al que se dirijan. Por todo ello, los cohetes de aire constituyen un excelente recurso práctico para favorecer la asimilación de los contenidos teóricos de la asignatura de Física.
\end{abstract}

Palabras clave: Cohete de aire; Lanzadera de pisotón; Medida de la velocidad; Tiro oblicuo.

Air rockets: construction, foundations and didactic applications for the study of Physics in high school and compulsory secondary education

Abstract: Although less known than water rockets, compressed air-driven rockets are simpler to build, they have a similar range and they do not present risks either on their handling nor on their launch because the energy needed to power them is obtained pressing a flexible container filled with air with the foot. The activities that can be carried out with these rockets can be adapted both for students of compulsory secondary education and for non-compulsory secondary education students and they allow multiple approaches, depending on the level of the students addressed. Therefore, air rockets are an excellent practical resource to promote the assimilation of the theoretical content from the Physics subject.

Keywords: Air rocket; Stompt shuttle; Speed measurement; Oblique launch.

Para citar este artículo: Tomás-Serrano A., Hurtado-Pérez J. (2019) Cohetes de aire: construcción, fundamentos y aplicaciones didácticas para el estudio de la Física en bachillerato y secundaria. Revista Eureka sobre Enseñanza y Divulgación de las Ciencias 16(3), 3401. doi: 10.25267/Rev_Eureka_ensen_divulg_cienc.2019.v16.i3.3401

\section{Introducción}

Los cohetes de aire son unos pequeños proyectiles, con forma de cohete (con un perfil que recuerda al de una flecha), que se impulsan mediante aire comprimido. Básicamente constan de un tubo con alerones en un extremo y un cono en el otro. Dado que pueden alcanzar una velocidad próxima a los $120 \mathrm{~km} / \mathrm{h}$, y son muy sencillos de fabricar, pueden constituir un excelente recurso para el estudio de contenidos básicos de física relacionados con cinemática (alcance máximo, velocidad, aceleración), dinámica (leyes de Newton, inercia, momento lineal) y energía (trabajo, energía cinética, energía potencial).

Para impulsar estos cohetes hay diferentes tipos de lanzaderas, desde un modelo que se comercializa como juguete infantil, hasta otros sistemas más complejos en los que, mediante 


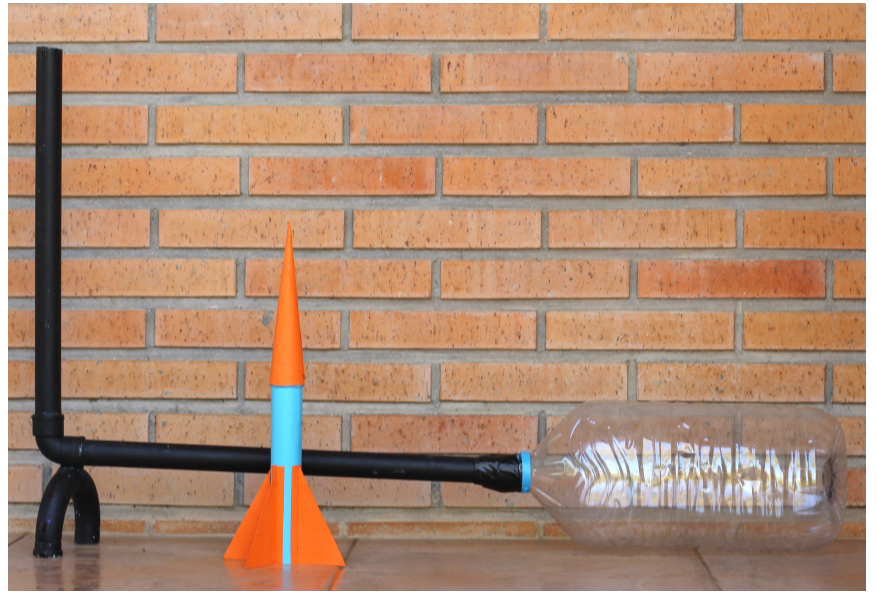

Figura 1. Lanzadera de pisotón construida con una garrafa de plástico y una tubería rígida de PVC. Junto a ella se muestra un cohete fabricado con cartulina. un conjunto de bomba, manómetro y válvulas, se puede incrementar a voluntad la presión de una cámara de aire, antes de efectuar el lanzamiento (Rønningen et al. 2012). En nuestra propuesta de trabajo hemos optado por la denominada lanzadera de pisotón, así llamada porque para lanzar el cohete hay que presionar bruscamente con el pie una cámara de aire. Como se aprecia en la figura 1, esta lanzadera está constituida por un recipiente flexible lleno de aire, a la presión atmosférica, y un tubo, en cuyo extremo libre se inserta el cohete.

\section{Construcción de la lanzadera y de los cohetes}

Tanto la lanzadera como los cohetes se pueden construir con materiales, de muy bajo coste, que los estudiantes pueden conseguir fácilmente en su entorno (Harrison 2019). Se precisará un recipiente de plástico, de una capacidad comprendida entre $3 \mathrm{~L}$ y $6 \mathrm{~L}$, que se pueda deformar fácilmente al presionarlo (p. ej., una garrafa PET vacía de agua), unos 2 metros de tubería rígida de $\mathrm{PVC}$ de unos $3 \mathrm{~cm}$ de diámetro, dividida en dos tramos de longitud similar (las tuberías flexibles no son adecuadas para este fin por su tendencia a curvarse), un codo de $90^{\circ}$ para conectar los dos tramos de tubería de la lanzadera, cinta adhesiva, cartulina y pegamento para papel.

Para construir la lanzadera habrá que ensamblar, mediante el codo de unión, los dos trozos de tubería de PVC; este conjunto se conecta a la boca del recipiente flexible de plástico. Utilizando masilla, cinta adhesiva u otro material similar se sellan las uniones (de la garrafa al tubo y de los tubos entre sî) para eliminar fugas de aire. Si no se utiliza adhesivo para unir los dos tramos de tubería, se podrá variar la orientación del segundo tramo para hacer lanzamientos tanto verticales como oblicuos (con diferente ángulo sobre la horizontal). Para que el cohete se mueva lo menos posible en el momento del lanzamiento es conveniente colocar algún tipo de apoyo (p. ej., una base de madera) entre el tramo de tubería horizontal y el suelo.

Los cohetes deben ser lo más aerodinámicos posible; se pueden construir con dos cartulinas de tamaño A4, una para el cuerpo del cohete y otra para el cono de fricción y las aletas estabilizadoras.

El cuerpo del cohete es un cilindro, cerrado por un extremo, que debe ajustar lo mejor posible en el tubo de la lanzadera, pero con un rozamiento pequeño. Esto se puede conseguir arrollando otra cartulina sobre el tubo y construyendo el cuerpo del cohete sobre el conjunto tubo-cartulina (figura 2).

Finalmente, para mejorar el comportamiento aerodinámico del cohete, se le colocará un cono de fricción en la parte delantera (o morro) y tres o cuatro aletas, distribuidas simétricamente, en la base, que tendrán como misión estabilizar la trayectoria (CNES 2018); para evitar que las aletas se deformen cuando el cohete está en movimiento, es conveniente utilizar cartulina más rígida que la usada para el cuerpo del cohete (o, simplemente, usar dos cartulinas ordinarias pegadas). 
Para lanzar el cohete bastará con introducirlo completamente, por la base, en el extremo libre de la lanzadera y golpear enérgicamente con el pie sobre el depósito que contiene el aire. Como estos recipientes tienen la base y el cuello más rígidos, el pisotón debe darse transversalmente en la parte central del recipiente.

Alternativamente, se puede lanzar el cohete dejando caer un objeto pesado (tal como una botella de plástico llena de arena) sobre la cámara de aire.

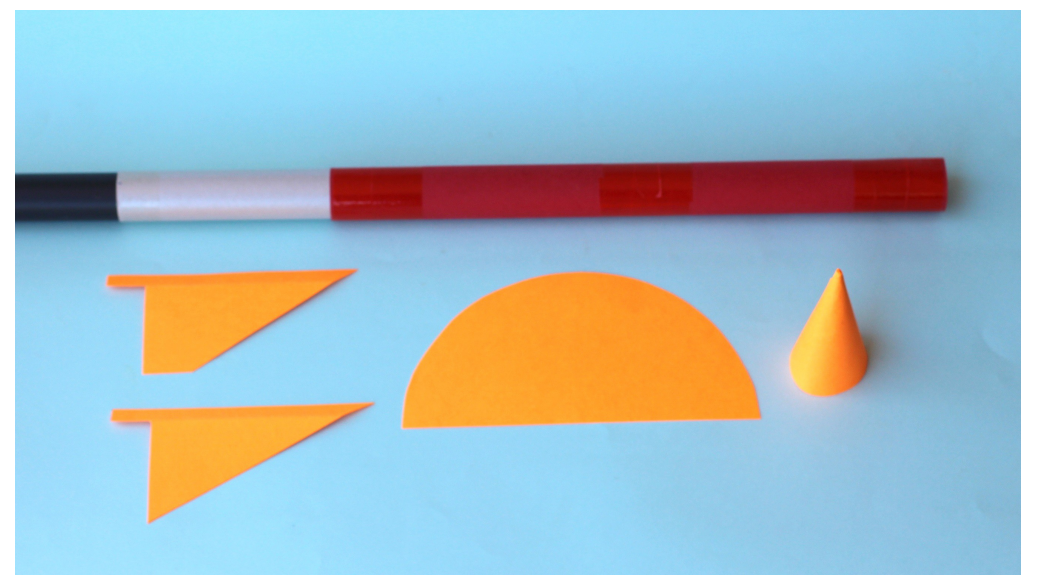

Figura 2. Detalle de la construcción del cohete. El cuerpo del cohete es un cilindro de cartulina arrollado sobre el tubo de la lanzadera; para conseguir un buen ajuste del cohete, es conveniente intercalar una segunda cartulina entre ambos. El cono de fricción se puede construir a partir de un semicírculo de cartulina; las aletas, de forma triangular o trapezoidal, son sencillas de construir y dan buen resultado.

\section{Precauciones y normas de seguridad}

Aunque el lanzamiento de este tipo de cohetes no presenta prácticamente ningún riesgo, deben respetarse algunas normas elementales de seguridad.

- En primer lugar, debe elegirse un emplazamiento al aire libre, preferiblemente en un día sin viento y lejos de cualquier zona por la que circulen personas o vehículos.

- Para evitar posibles caídas, la persona que da el pisotón debe estar apoyada en otra. Por la misma razón, no es aconsejable saltar sobre la lanzadera.

- La zona próxima a la lanzadera debe estar completamente despejada para que el cohete no pueda golpear accidentalmente a nada ni a nadie.

\section{Fundamento}

Cuando se presiona rápidamente la cámara de aire de la lanzadera, se provoca en ella una perturbación causada por el súbito aumento de presión. Esta perturbación se propaga por la cámara a la velocidad del sonido y ejerce una fuerza sobre el cuerpo del proyectil que, insertado en el extremo del tubo de la lanzadera, cierra la cámara de aire (Resnick, Halliday y Krane 2002). La aceleración debida a esta fuerza es tal que el proyectil sale de la lanzadera a una velocidad que puede superar los $100 \mathrm{~km} / \mathrm{h}$, tal y como se calcula a continuación.

Si llamamos $\Delta p$ al valor medio de la presión que se origina, $S$ a la sección transversal del proyectil, $m$ a su masa y $f$ a la resultante de las fuerzas de rozamiento que afectan al cohete (con el tubo de la lanzadera y con el aire), la fuerza neta media $F$ que actúa sobre el cohete será

$$
F=\Delta p \cdot S-m \cdot g-f,
$$

siendo $g$ la aceleración de la gravedad. 
A partir de la ecuación (1) y estableciendo algunas aproximaciones (NASA 2018), podemos hacer una estimación de la velocidad inicial $v$ del cohete.

En primer lugar, consideraremos que la presión (y, por tanto, la fuerza) que impulsa el cohete permanece prácticamente constante durante el tiempo que dura el lanzamiento, esto es, mientras el cohete recorre la distancia $d$, que está insertado en el tubo de la lanzadera, sometido a la fuerza $F$ que lo impulsa; como se observa en la figura 3, esta distancia es igual a la longitud total del cohete, exceptuando el cono de fricción.

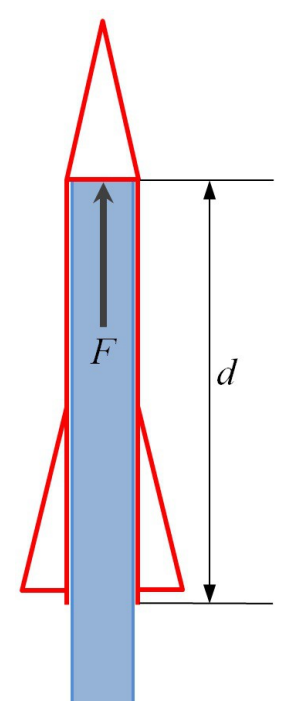

Figura 3. La fuerza $F$ que Sustituyendo los valores correspondientes a un cohete de cartulina impulsa el cohete se puede considerar constante mientras el cohete recorre una distancia $d$, que es igual a la la distancia que está insertado en el tubo de la lanzadera. $10 \%$ mayor de lo predicho por la ecuación (2), ya que el aire que sale de la cámara, tras el pisotón, también empuja al cohete (NASA 2018). Podemos establecer una cota superior para esta energía adicional suponiendo que el aire sale a la misma velocidad que el cohete y le transfiere toda su energía. Si suponemos que la cámara de aire tiene una capacidad de $5 \mathrm{~L}$ y sale, aproximadamente, la mitad del aire como consecuencia del pisotón, la energía cinética $E_{\mathrm{c}}$ que puede aportar este aire al cohete será:

$$
E_{\mathrm{c}}=\frac{1}{2} m v^{2}=\frac{1}{2} 2,5 \times 1,2 \times 10^{-3} \times 27^{2}=1,1 \mathrm{~J},
$$

donde se ha tomado $1,2 \mathrm{~g} / \mathrm{L}$ como densidad del aire, a $20{ }^{\circ} \mathrm{C}$. Esta energía supondría un incremento de velocidad comprendido entre $2 \mathrm{~m} / \mathrm{s}$ y $3 \mathrm{~m} / \mathrm{s}$ para un cohete de unos $20 \mathrm{~g}$ de masa.

Como se verá más adelante, las medidas obtenidas experimentalmente para la velocidad de salida del cohete son compatibles con las deducidas mediante esta estimación.

\section{Medida de la presión generada en la lanzadera}

$\mathrm{El}$ aumento de presión $\Delta p$ que se genera en el interior de la lanzadera se puede medir con un sensor digital de presión (y el correspondiente software), similar al mostrado en la parte superior de la figura 4. 
Para efectuar la medida hay que añadir, mediante una derivación en T o Y, un segundo tubo al tramo horizontal de la lanzadera (figura 5). En uno de los tubos se acopla el cohete y en otro el sensor. Operando así se comprueba que el pico de presión está comprendido normalmente entre $2 \times 10^{4} \mathrm{~Pa}$ y $4 \times 10^{4} \mathrm{~Pa}$, dependiendo de las características de la cámara de aire y de cómo se presione.
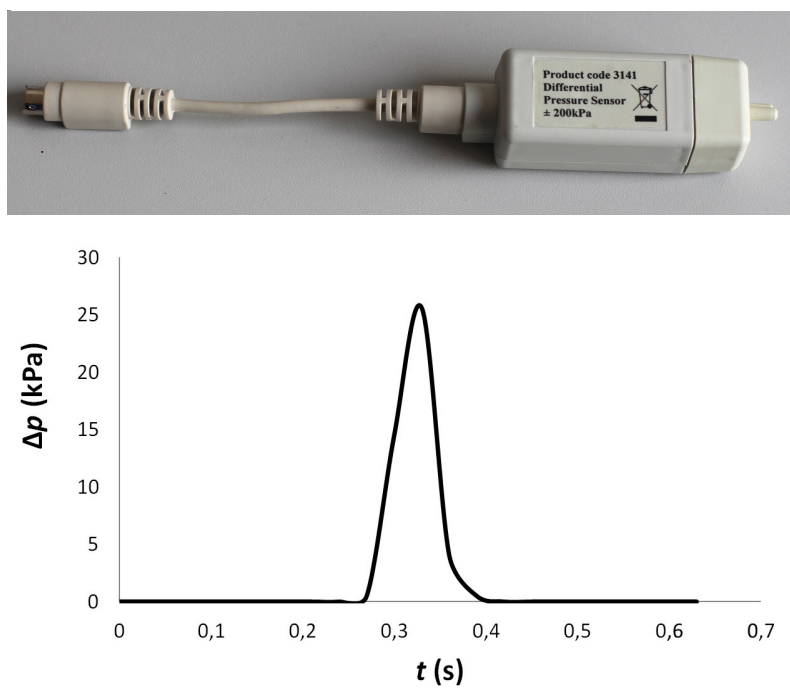

Figura 4. (Arriba) Fotografía del sensor digital de presión diferencial utilizado en este experimento (Data Harvest, \pm $200 \mathrm{kPa}$ ). (Abajo) Pulso de presión originado al dar el pisotón en la cámara de aire de la lanzadera. Aunque el pulso tiene una duración algo mayor de $0,1 \mathrm{~s}$, el cohete abandona la lanzadera en menos de $0,04 \mathrm{~s}$ (tal y como se deduce del visionado de las grabaciones en vídeo).

Si no se dispone de un sensor digital, se puede utilizar un manómetro para balones, con su aguja insertada en un tapón de corcho o caucho (figura 5-dcha.).

Como el pulso de presión es muy breve (su duración es aproximadamente de $0,1 \mathrm{~s}$ ), para poder apreciar el valor de la presión hay que grabar un vídeo del manómetro en el momento de dar el pisotón y visionarlo posteriormente fotograma a fotograma.

\section{Determinación de la velocidad inicial del cohete}

La velocidad de salida del cohete se puede medir, con un buen grado de precisión, a partir de una grabación en vídeo del lanzamiento del cohete. Básicamente, se trata de usar la grabación para medir la distancia que recorre el proyectil en el intervalo de tiempo correspondiente a un fotograma (Gil 2014, Vera, Rivera, Fuentes y Romero 2015).

Como la velocidad inicial de los cohetes puede superar ampliamente los $100 \mathrm{~km} / \mathrm{h}$, la grabación debe hacerse con una cámara de vídeo o de fotos (réflex o compacta) en la que se pueda fijar un tiempo de exposición para cada fotograma lo suficientemente pequeño para congelar el movimiento y evitar una imagen borrosa del cohete.
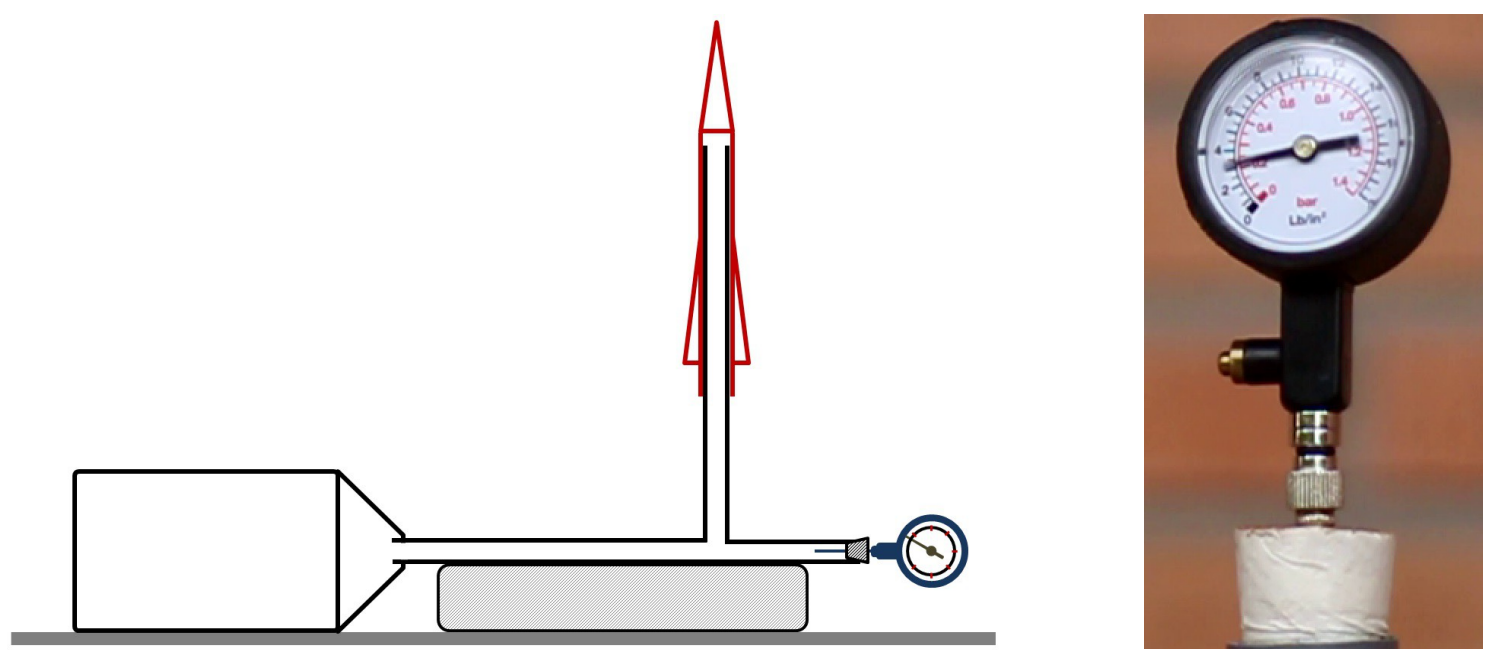

Figura 5. (Izqda.) El manómetro colocado en la rama horizontal mide la sobrepresión que se origina en la lanzadera en el momento en que se dispara el cohete. (Dcha.) captura de un fotograma de vídeo en el que se aprecia la indicación del manómetro en el momento del disparo. 
También se necesitará algún programa de edición de vídeo que permita visualizar la grabación fotograma a fotograma. Aunque en Internet hay varios programas de libre acceso y de fácil uso, el más cómodo de utilizar es Tracker, un programa muy intuitivo de análisis de vídeo y construcción de modelos físicos que está diseñado para ser usado en la enseñanza de la Física (Brown 2016). Con otros programas de edición de vídeo (tales como Avidemux, VirtualDub, etc.) se pueden obtener resultados similares, pero el proceso es más engorroso.

Veamos con algo más de detalle el procedimiento a seguir para determinar la velocidad inicial del cohete utilizando Tracker.

Necesitaremos una grabación, con la mayor calidad de imagen posible, del momento del lanzamiento del proyectil. Como para hallar la velocidad de salida del cohete solo se necesitan los primeros fotogramas, inmediatamente posteriores al lanzamiento, el aparato de grabación debe estar situado a una distancia adecuada de la lanzadera para que se graben, al menos, los primeros 150 centímetros del ascenso del cohete. Además es conveniente utilizar un trípode para evitar vibraciones que perjudicarían la calidad de las imágenes.

Para medir la distancia que recorre el cohete se puede tomar como referencia la longitud del propio cohete o bien, colocar la lanzadera próxima a una pared y marcar sobre ella una distancia conocida que nos sirva para determinar la posición del cohete a medida que asciende.

En cuanto a la determinación de la velocidad inicial $v$ del proyectil, se necesita conocer la distancia $\Delta s$ que recorre en un intervalo de tiempo $\Delta t$ muy pequeño. La distancia se obtendrá comparando la posición del cohete en dos fotogramas sucesivos y el tiempo vendrá dado por la velocidad de grabación de la cámara de vídeo. Estas medidas son las que pueden realizarse rápidamente con el programa Tracker, mediante las herramientas denominadas Calibration Stick y Video/Frame Durations.

Como ejemplo, se muestran en la figura 6 dos fotogramas sucesivos de una grabación de vídeo, efectuada a 50 fotogramas por segundo $(\Delta t=1 / 50 \mathrm{~s}=0,020 \mathrm{~s})$ del lanzamiento de un cohete de $20 \mathrm{~g}$ de masa y $8 \mathrm{~cm}^{2}$ de sección. Tomando como referencia la longitud del propio cohete $(0,410 \mathrm{~m})$, el programa determina que la distancia $\Delta s$ recorrida por el cohete en el intervalo de tiempo $\Delta t$ transcurrido entre esos dos fotogramas es $0,641 \mathrm{~m}$, por lo que la velocidad media $v_{\mathrm{m}}$ del cohete en este intervalo de tiempo será $v_{\mathrm{m}}=0,641 / 0,020=32 \mathrm{~m} / \mathrm{s}$ (115 km/h).

Dado que el intervalo de tiempo correspondiente a un fotograma es suficientemente pequeño (de $0,042 \mathrm{~s}$ a $0,020 \mathrm{~s}$ para las velocidades de grabación de las cámaras de vídeo ordinarias), podemos admitir que la velocidad inicial $v$ del cohete estará muy próxima al valor obtenido para $v_{\mathrm{m}}$.

De acuerdo con la ecuación (2), la velocidad inicial del cohete debe aumentar al hacerlo la relación sección/masa, $S / \mathrm{m}$. Con este método se puede comprobar que, efectivamente, esto ocurre así; de hecho, los cohetes que tienen la mayor relación $S / m$ pueden superar los $30 \mathrm{~m} / \mathrm{s}$. No obstante, cuando se comparan los alcances de los cohetes (tanto en trayectoria vertical como parabólica), se observa que son similares, debido al rozamiento con el aire, que aumenta con la sección del cohete y el cuadrado de la velocidad (Widmark 1998).

\section{Balance energético}

Aunque las lanzaderas de pisotón logran impulsar los cohetes a velocidades que, generalmente, superan los $100 \mathrm{~km} / \mathrm{h}$, su eficiencia energética es modesta. 

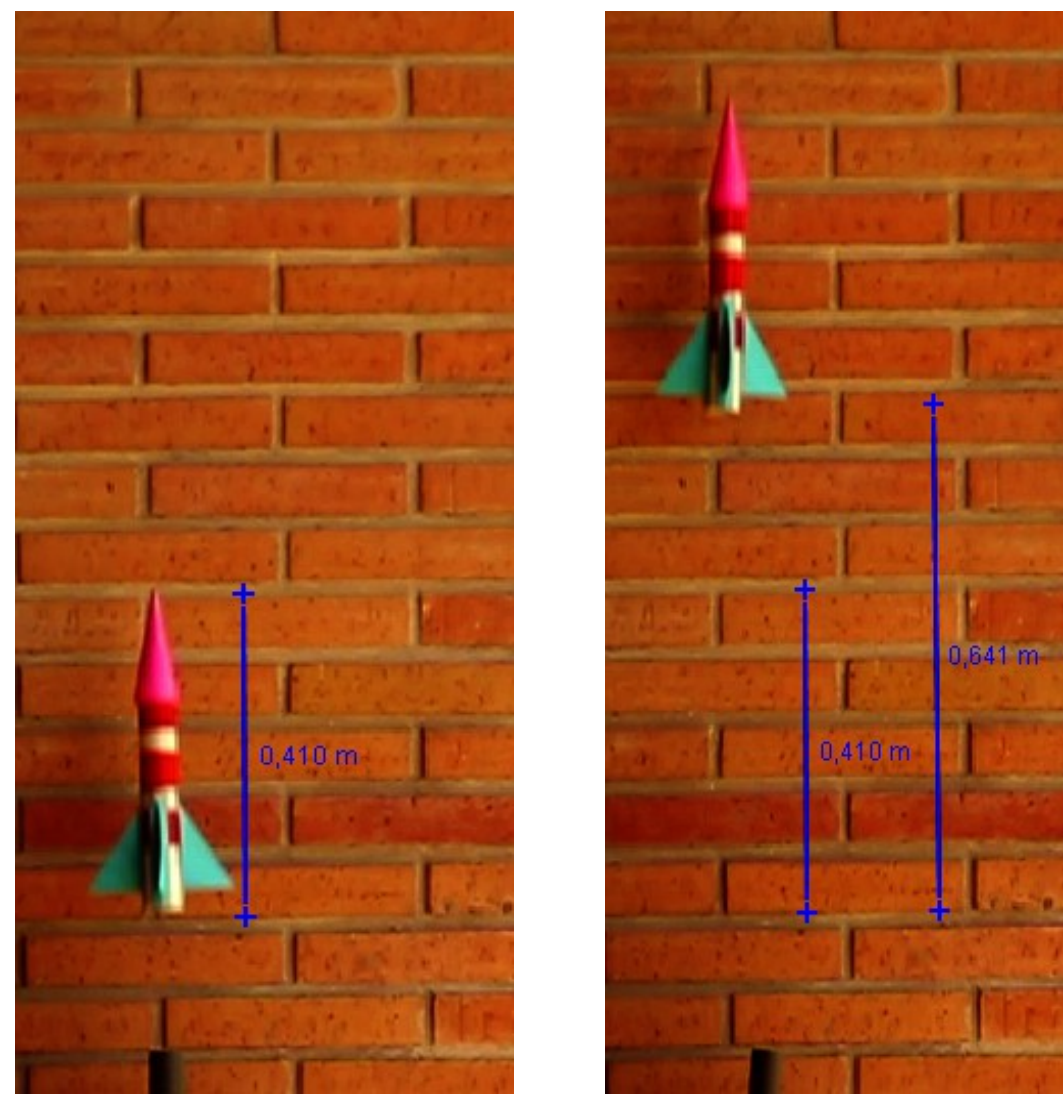

Figura 6. Imágenes de dos fotogramas sucesivos del vídeo (separados por un intervalo de $0,020 \mathrm{~s}$ ), grabado a $50 \mathrm{fps}$, correspondiente al lanzamiento de un cohete. Con la herramienta de Tracker denominada Calibration Stick se puede establecer una correlación entre los valores reales de las distancias y las correspondientes medidas sobre los fotogramas. A partir de estos valores y sabiendo que el tiempo transcurrido entre estas dos imágenes es $0,020 \mathrm{~s}$, es inmediato el cálculo de la velocidad del cohete.

Una forma sencilla de medir esta eficiencia consiste en dejar caer un objeto de masa $M$, desde una altura $H$ conocida, sobre la cámara de aire (en lugar de presionar con el pie), de modo que la energía aportada a la lanzadera es $M \cdot g \cdot H$. Si se conoce la masa $m$ del cohete y su velocidad inicial $v$, entonces el rendimiento energético de la lanzadera vendrá dado por el cociente entre la energía cinética de salida del cohete y la energía potencial inicial de la masa $M$.

Experimentalmente se encuentra que si se deja caer una masa $M$ de $7 \mathrm{~kg}$, desde una altura $H$ de $70 \mathrm{~cm}$, sobre una lanzadera similar a la mostrada en la figura 1 , un cohete de $20 \mathrm{~g}$ de masa $m$ (como el mostrado en la figura 6) alcanza una velocidad máxima $v$ de unos $32 \mathrm{~m} / \mathrm{s}$, por lo que el rendimiento de este sistema de lanzamiento está normalmente por debajo del $25 \%$ :

$\frac{1}{2} m v^{2} / M g H=\left(\frac{1}{2} 20 \times 10^{-3} \times 32^{2} \mathrm{~J}\right) /(7 \times 9,8 \times 0,7 \mathrm{~J})=0,21$.

La mayor parte de la energía aportada por el pisotón, o por el objeto que se deja caer sobre la cámara de aire, se invierte en la producción de calor y de deformaciones. 


\section{Algunos planteamientos didácticos de esta actividad}

Todas las actividades relacionadas con el diseño, construcción y lanzamiento de los cohetes de aire tienen una excelente acogida entre el alumnado de secundaria y bachillerato.

Dependiendo del nivel educativo al que se dirija, la actividad puede tener diferentes enfoques. En los primeros cursos de secundaria se puede plantear como una actividad lúdica (figura 7) o como una pequeña investigación encaminada a mejorar la estabilidad de la trayectoria de los cohetes. En este segundo caso, se podría comenzar con un simple tubo de cartulina, cerrado por un extremo, al que, posteriormente, se le acoplarían varillas largas (como los cohetes de fuegos artificiales), aletas de diferente tamaño y forma (en diferente número y posición) y distintos tipos de morro (plano, cónico o semiesférico).

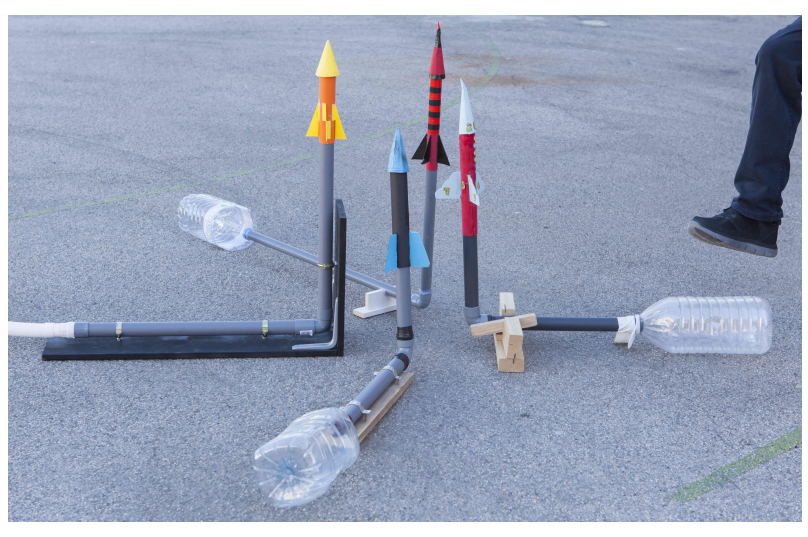

Figura 7. Fotografía de lanzaderas de cohetes fabricadas por alumnos de secundaria. tratará de analizar experimentalmente cómo influye, sobre la velocidad inicial de los cohetes, el valor de la intensidad del pulso de presión $(\Delta p)$, la relación sección/masa $(S / \mathrm{m})$ de los cohetes y la distancia $(d)$ que recorren sobre el tubo de la lanzadera. Dado que el alcance vertical es más difícil de medir con precisión, resulta más sencillo variar la orientación del tubo de la lanzadera para efectuar lanzamientos oblicuos y medir el alcance horizontal.

Otro enfoque complementario para bachillerato puede ser el empleo de la lanzadera para estudiar el tiro oblicuo omitiendo, en principio, los efectos del rozamiento del aire, ya que su consideración queda fuera del alcance de los contenidos de esta etapa. En concreto, se podrían llevar a cabo sendas investigaciones acerca de cómo varía el alcance de los proyectiles con la velocidad inicial del cohete y con el ángulo de lanzamiento. En todo caso, será altamente instructivo para los estudiantes el comprobar que el alcance real del cohete, para unos valores dados del ángulo y velocidad de salida, está en torno al $50 \%$ del valor calculado sin tener en cuenta el rozamiento con el aire.

Para llevar a cabo estos estudios, en lugar de utilizar la técnica del pisotón, los cohetes deben impulsarse dejando caer sobre la cámara de aire, desde diferentes alturas, un objeto de una masa comprendida entre $5 \mathrm{~kg}$ y $7 \mathrm{~kg}$.

\section{Conclusiones}

Hay muchos recursos experimentales que logran atraer la atención del alumnado, pero muy pocos lo consiguen de forma tan efectiva como los cohetes de aire (Martin 1969). Por otra parte, el hecho de que los estudiantes deban usar tecnología informática para abordar determinados aspectos de las actividades que aquí se plantean (tal como ocurre con la medida 
de la velocidad de salida de los cohetes) influye directamente en que se impliquen más en un proyecto que, como el presente, requiere altos niveles de actividad. $\mathrm{Y}$ es sabido que un incremento de la motivación del alumnado puede conseguir un aprendizaje más significativo (Pedró 2015).

Aunque hay diferentes tipos de lanzaderas para estos cohetes, nosotros hemos optado por un modelo muy simple, porque se puede construir en poco tiempo, se emplean materiales de bajo coste y tanto la construcción de la lanzadera como su uso están prácticamente exentos de riesgos si se siguen unas normas básicas de seguridad.

Las actividades relacionadas con los cohetes de aire se pueden adaptar fácilmente para alumnos de diferentes etapas educativas, desde bachillerato hasta secundaria obligatoria e, incluso, para alumnos de los últimos cursos de primaria.

Dependiendo de los contenidos que se deseen abordar, se puede plantear tanto una actividad recreativa como un trabajo de investigación en el que, sin necesidad de utilizar materiales convencionales de laboratorio, se pueden hacer medidas cuantitativas (Gil, Calderón, Núñez, Laccio y Iannelli 2014) y determinar parámetros tales como la variación de presión que impulsa al cohete, la velocidad de salida o el rendimiento energético de este sistema de lanzamiento de proyectiles.

Con el método del pisotón es muy difícil controlar la velocidad de salida de los proyectiles. Pero si en lugar de presionar con el pie la cámara de aire, se deja caer sobre ella un objeto pesado, se podrá modificar con bastante fiabilidad dicha velocidad; bastará con variar la masa y/o la altura del objeto que se deja caer sobre la cámara de aire de la lanzadera.

Por otra parte, y a pesar de los efectos del rozamiento con el aire, las medidas de la velocidad inicial de los cohetes se pueden correlacionar, de manera aceptable, con los resultados obtenidos para el alcance máximo a diferentes ángulos de lanzamiento.

\section{Referencias}

Brown D. (2019) Tracker. Video Analysis and Modeling Tool.

CNES (2008) Le Vol de la Fusée, Stabilite et Trajectographie. París: Centre National d'Études Spatiales.

Gil S. (2014) Experimentos de Física usando las TIC y elementos de bajo costo. Buenos Aires: Alfaomega.

Gil S., Calderón S., Núñez P., Di Laccio J., Iannelli L. (2014) Aulas-laboratorios de bajo costo, usando TIC. Revista Eureka sobre Enseñanza y Divulgación de las Ciencias 12(1), 212-226.

Harrison S. (2019) How to make air rockets. Williamsport (Pennsylvania): SciencetoyMaker.

Martin W. M. (1969) Air-Rockets. The Physics Teacher 7, 453-454.

NASA (2018) Air Rocket Launch. Whasington: National Aeronautics and Space Administration.

Pedró F. (2015). Tecnología para la mejora de la educación. Documento básico. Madrid: Fundación Santillana.

Resnick R., Halliday D., Krane K. (2002) Física, $4^{a}$ ed. México: CECSA.

Rønningen J. E., Sheth R., Vestnes F., Råken M. (2012) Sky-high science: building rockets at school. Science in School. The European Journal for Science Teachers 22, 36-41. 
Vera Mathias F., Rivera Campos R., Fuentes R., Romero Maltrana D. (2015) Estudio del movimiento de caída libre usando vídeos de experimentos. Revista Eureka sobre Enseñanza y Divulgación de las Ciencias 12(3), 581-592.

Widmark S. A. (1998) Rocket physics. The Physics Teacher 36, 148-153. 